\title{
EL CONTENIDO DEL VAPOR DE AGUA DE LA ATMÓSFERA COMO INDICADOR DEL CALENTAMIENTO GLOBAL EN UNA ZONA DEL CARIBE DE COLOMBIA
}

\author{
M. PÉREZ-VILORIA, L. GÓNIMA* \\ Grupo de Materiales y Física Aplicada. Departamento de Física y Electrónica. Universidad de Córdoba. \\ Cra. 6 No. 76-103. Montería, Colombia.
}

RESUMEN. Este estudio muestra la importancia del contenido de vapor de agua atmosférico como indicador del calentamiento global a escala local, en parte de la cuenca media y baja del río Sinú (Departamento de Córdoba, Colombia). El régimen climático de la zona seleccionada está representado por las estaciones meteorológicas de Turipaná, El Salado, Lorica y La Doctrina. Series de datos (promedios mensuales) de la temperatura del aire $T$ y la humedad relativa $H R$ de las estaciones mencionadas, entre 1968 y 2011, fueron seleccionadas y sometidas a rigurosos análisis estadísticos y climáticos para evaluar su calidad. Posteriormente, se estimaron datos de la densidad de vapor de agua atmosférica $d_{v}$ utilizando promedios anuales de T y HR previamente calculados para cada uno de los 44 años seleccionados. El análisis de tendencia interanual de los promedios anuales de $T$ y $d_{v}$ para las cuatro estaciones seleccionadas y la zona de estudio integrada demuestra que, aunque la variabilidad climática de dicha zona depende estrechamente de los fenómenos El Niño y La Niña, la tendencia al aumento de ambas variables se mantiene desde 1968. En particular, en la zona de estudio integrada se detectó un incremento en $T$ de $0.75^{\circ} \mathrm{C}(2.7 \%)$ ó $0.17^{\circ} \mathrm{C}$ dec $^{-1}$, valor que coincide con el reportado por la OMM a nivel mundial, mientras que $d_{v}$ aumentó en $1.02 \mathrm{~g} \mathrm{~m}^{-3}(5.9 \%)$ equivalente a $0.23 \mathrm{~g} \mathrm{~m}^{-3} \mathrm{dec}^{-1}$. Asimismo se determinó que 1991-2000 fue la década más cálida del período 1968-2011 y no la siguiente (2001-2010), como se expone en varios estudios realizados a nivel mundial. Contrariamente a los resultados obtenidos en investigaciones sobre el clima global y regional, en este estudio los promedios decadales no explican completamente la gran variabilidad climática tropical local y el efecto producido por el fenómeno del calentamiento global. El análisis estadístico realizado para las tendencias de $T$ y $d_{v}$ en los períodos de tiempo 1968-1974 y 1975-2011 permitió identificar evidencias del posible inicio del calentamiento global en la zona de estudio, debido probablemente al más intenso evento La Niña de la historia, registrado entre 1973 y 1974 (ONI de -2.0). Los resultados obtenidos sobre el calentamiento global en las cuatro estaciones seleccionadas y en la zona de estudio integrada, causado posiblemente por la extensa e intensa intervención antrópica 
de dicha zona, confirman que el contenido de vapor de agua atmosférico d es la variable termodinámica más adecuada para la descripción del comportamiento del régimen climático tropical a escala local.

\section{Atmospheric water vapor content as indicator of global warming in a Caribbean zone of Colombia}

ABSTRACT. This study shows the importance of the atmospheric water vapor content as an indicator of global warming at local scale in part of the middle and lower basin of the Sinu river (Colombia). The climatic regime of the selected zone is represented by the meteorological stations of Turipaná, El Salado, Lorica and La Doctrina. Data series of air temperature T and relative humidity HR of the mentioned stations, between 1968 and 2011, were selected and tested for quality by rigorous statistical and climatic analysis. Furthermore, atmospheric water vapor density data $d_{v}$ were estimated, using mean annual values of $T$ and HR previously calculated for each of the selected 44 years. The interannual trend analysis of the annual means of $T$ and $d$, for the four selected stations and the integrated studied zone, demonstrated that even though their climatic variability strongly depends of El Niño and La Niña phenomena, the upward trend of both variables remains since 1968. Particularly in the integrated studied zone, an increment in $T$ of $0.75^{\circ} \mathrm{C}(2.7 \%)$ or $0.17^{\circ} \mathrm{C} \mathrm{dec^{-1 } \text { was }}$ detected, which coincides with the reported global value by WMO, while $d_{v}$ increased in $1.02 \mathrm{~g} \mathrm{~m}^{-3}(5.9 \%)$ or $0.23 \mathrm{~g} \mathrm{~m}^{-3} \mathrm{dec}^{-1}$. It was determinated likewise that 1991-2000 was the warmest decade of the period of time 1968-2011 and not the next one (20012010), as it is exposed in diverse studies worldwide. On the contrary of the results obtained from global and regional research, in this study the decadal means do not explain completely the great tropical climatic variability at local level and the effect of the global warming. The statistical analysis of $T$ and $d_{v}$ trends for the periods of time 1968-1974 and 1975-2011 allowed to identify evidences of the possible beginning of the global warming in the studied zone, probably due to the most intensive La Niña event of the history, registered between 1973 and 1974 (ONI of -2.0). The results obtained in this study about the global warming in the four selected meteorological stations and the integrated studied zone, probably caused by extense and intense human land intervention, confirm that the atmospheric water vapor content $d_{v}$ is the most adequate thermodynamic variable for the description of the behavior of tropical climatic regimes at local level.

Palabras clave: variaciones climáticas, temperatura del aire, contenido de vapor de agua atmosférico, calentamiento global, Colombia.

Key words: climatic variations, air temperature, atmospheric water vapor content, global warming, Colombia.

Enviado el 8 de julio de 2013

Aceptado el 12 de diciembre de 2013

* Correspondencia: Departamento de Física y Electrónica. Universidad de Córdoba. Cra. 6 No.76-103. Montería, Colombia. E-mail: lgonima@correo.unicordoba.edu.co 


\section{Introducción}

El calentamiento global que actualmente está ocurriendo en nuestro Planeta, como consecuencia de la modificación de la composición de la atmósfera y por ende del ambiente terrestre, está directamente relacionado con la actividad humana desarrollada principalmente durante las últimas 6 décadas (UNFCCC, 1992), la cual está generando cada vez mayores efectos negativos sobre la Naturaleza (OMM, 2008).

Para responder a esta situación desde el punto de vista científico, la Organización Meteorológica Mundial (OMM) y el Programa de las Naciones Unidas para el Medio Ambiente (PNUMA) han definido que las variables meteorológicas generadoras del calentamiento global, están representadas por el incremento de la temperatura del aire, variaciones en el contenido de humedad del aire, cambios en los regímenes de lluvias y el incremento del nivel del mar (IPCC, 2001). Un ejemplo de estudios realizados sobre este tema es el de Aro (1976), en el que se calculó la densidad de vapor de agua a nivel de la superficie a partir de datos de la humedad relativa, con el fin de compararla estadísticamente con el contenido de vapor de agua de la atmósfera determinado a través de radiosondeos atmosféricos. Moeller-Houborg y Soegaard (2004) determinaron de flujos de $\mathrm{CO}_{2}$ y de vapor de agua con datos de satélite, para analizar los ecosistemas de Dinamarca. Dai (2006), a partir de mediciones de la temperatura del aire, temperatura del punto de rocío y presión atmosférica (1975-2005) de la zona geográfica comprendida entre los $60^{\circ} \mathrm{S}$ y $75^{\circ} \mathrm{N}$ del globo terráqueo, calculó las humedades específica y relativa relacionándolas con variaciones interanuales y estacionales de la temperatura del aire y con simulaciones obtenidas mediante el modelo climático PCM. Seidel et al. (2007) analizaron en su totalidad y por décadas las tendencias del punto de rocío, la temperatura del aire, la razón de mezcla y la humedad relativa, para el estudio del clima del Monte Washington (Estados Unidos) entre 1935 y 2004. Murphy et al. (2010), basándose en el análisis del calentamiento global a escala mundial, plantean una discusión sobre el desafío que representa actualmente para la ciencia del clima, la creciente importancia que está adquiriendo el análisis y la predicción de variaciones climáticas por medio de promedios decadales. Kousary et al. (2011) analizaron, durante un periodo de 55 años, las tendencias de la temperatura del aire, la precipitación y la humedad relativa, para identificar variaciones del clima en las regiones Este y Central de Irán. Por otra parte, Isaac y van Wijngaarden (2012) analizando detenidamente la homogeneidad de datos de la temperatura del aire y la humedad relativa de 309 estaciones meteorológicas situadas a lo largo y ancho de Norteamérica, entre 1948 y 2010, calcularon la presión de vapor de agua y determinaron posteriormente sus tendencias, encontrando que estas últimas muestran una relación estadísticamente significativa con el calentamiento global que experimentó parte de Estados Unidos, Canadá y el Ártico occidental durante el período de tiempo estudiado. Recientemente, Willett et al. (2013) utilizaron la base de datos HadCRUH, para la descripción y estudio del contenido de humedad de la superficie a nivel global.

En Colombia se han realizado varias investigaciones sobre el calentamiento global y sus impactos, con el propósito de establecer medidas de adaptación y mitigación frente a los efectos que produce este fenómeno. Así, cabe citar el trabajo de León-Aristizabal 
(2000), en el que se evaluaron las series mensuales de la temperatura del aire de todo el país, para determinar las tendencias de dicha variable y compararlas con las respectivas del IPCC a nivel mundial. Ruiz-Murcia (2010), utilizando modelos climáticos globales y regionales, analizó series de precipitación, temperatura del aire y humedad relativa, entre 1971 y 2000, detectando evidencias del calentamiento global en Colombia, mientras que Benavides-Ballesteros y Rocha-Enciso (2012) evaluaron el comportamiento temporal por décadas de la temperatura del aire y la precipitación en el territorio colombiano, entre 1961 y 2011, para después generar indicadores de calentamiento global para todo el país. Finalmente, Ocampo-López (2012) analizó estadísticamente series de datos meteorológicas (1981-2010), para identificar algunas señales del calentamiento global (aumento de la temperatura media del aire e incremento en la frecuencia de las precipitaciones extremas) en la cuenca del río Chinchiná (Departamento de Caldas).

A nivel regional (Departamento de Córdoba), Gónima (2009) muestra evidencias del calentamiento global en el Caribe colombiano, representadas por variaciones de la temperatura del aire, la precipitación, la evapotranspiración y el déficit hídrico. Por otra parte, Padilla-Agámez (2011) analizó el efecto del calentamiento global (variaciones de la temperatura del aire, las precipitaciones y el déficit hídrico) sobre la producción agrícola del cultivo de arroz en el Distrito de Riego de la Doctrina. Recientemente, PérezViloria (2012) presenta nuevas evidencias del calentamiento global en el Caribe colombiano, mediante la comparación de los resultados obtenidos del análisis de tendencias (1962-2011) de variables meteorológicas medidas y calculadas (temperatura del aire, humedad relativa, tensión y densidad del vapor de agua atmosféricos).

En concordancia con lo expuesto anteriormente, el presente trabajo muestra la importancia que tiene el contenido de vapor de agua atmosférico, la densidad de vapor de agua, como indicador del calentamiento global a nivel local (parte de la cuenca media y baja del río Sinú-Departamento de Córdoba), entre 1968 y 2011.

\section{Zona de estudio}

La zona de estudio seleccionada (Fig. 1), que incluye gran parte de la cuenca media y baja del río Sinú, está conformada por parte de los municipios de Montería, San Carlos, Cereté, San Pelayo, Ciénaga de Oro, Cotorra, Chimá, Momil, Purísima y Lorica en el Departamento de Córdoba (IGAC, 2009) y cubre un área aproximada de 2800 km², plana en general y con una altitud media de 20 m s.n.m. El régimen climático, representado por las estaciones meteorológicas de Turipaná, El Salado, Lorica y La Doctrina (IDEAM, 2005; Ruiz-Murcia, 2010), está caracterizado por una temperatura media y una precipitación anuales de $27.5^{\circ} \mathrm{C}$ y $1300 \mathrm{~mm}$ respectivamente (CVS et al., 2006). Según el Plan de Ordenamiento y Manejo Integral de la Cuenca Hidrográfica del río Sinú (POMCA-RS), la red hídrica está constituida principalmente por el río Sinú, que atraviesa el Departamento de sur a norte y por un sistema de ciénagas, altamente intervenidas, distribuidas a lo largo de su cauce. La cobertura vegetal está representada por pastos, cultivos y algunas áreas con bosques y vegetación ribereña asociada a las orillas del mencionado río y el suelo es utilizado para la actividad agrícola y la ganadería extensiva (CVS et al., 2006). 


\section{Metodología}

\subsection{Adquisición y evaluación de datos meteorológicos}

La información meteorológica básica utilizada para el desarrollo de este estudio, estuvo representada por series de datos (promedios mensuales) de la temperatura del aire T y de la humedad relativa HR, correspondientes al periodo de tiempo entre 1968 y 2011 (44 años). Esta información fue suministrada por el Instituto de Hidrología, Meteorología y Estudios Ambientales (IDEAM), previamente evaluada para detectar errores causados por los instrumentos de medida, la observación y la transmisión de los datos. De las seis estaciones meteorológicas con series de datos disponibles en la zona de estudio seleccionada, se escogieron cuatro (Fig. 1), que fueron aquellas con la mayor longitud temporal (> 30 años). A continuación y como condición inicial para verificar si las estaciones meteorológicas seleccionadas podrían ser agrupadas por medio de una regionalización climática estacional, se procedió a analizar el comportamiento de los promedios mensuales multianuales calculados de $\mathrm{T}$ (Fig. 2), comprobándose una gran semejanza entre ellos para las cuatro estaciones. Luego y con base en los promedios anuales calculados de T, para cada uno de los 44 años seleccionados, se observó también una gran similitud en la variabilidad climática de las series de datos de cada una de las estaciones (Fig. 3).

El mismo procedimiento se siguió con HR, verificándose que, aunque sus variaciones mensuales siguen una tendencia climática similar para las cuatro estaciones (Fig. 2), las respectivas variaciones interanuales son muy pequeñas (Tabla 1) y no presentan patrones definidos, lo cual concuerda con lo expuesto en Ruiz-Murcia (2010) para Colombia.

Tabla 1. Variación interanual de la humedad relativa HR (\%) para cada una de las estaciones meteorológicas (1968-2011).

\begin{tabular}{|c|c|c|c|c|}
\hline Estación & Turipaná & El Salado & Lorica & La Doctrina \\
\hline Variación & $80-83$ & $79-87$ & $82-86$ & $82-87$ \\
\hline Promedio & 81 & 83 & 84 & 85 \\
\hline
\end{tabular}

Una vez identificado el régimen climático de las variables mencionadas, las series de datos originales de T y HR fueron sometidas a procesos estadísticos, con el fin de evaluar y asegurar una adecuada calidad de ellas, de acuerdo con la siguiente metodología (Fernández-García, 1995; Wilks, 2006; WMO, 2011): realización de un análisis básico de los datos representado por el cálculo de valores medios, diferencias y desviaciones estándar con el fin de determinar la ocurrencia de datos atípicos, cálculo y análisis de rachas para evaluación de la aleatoriedad de los datos, test de persistencia (R) para determinación de la independencia de los datos y tests $\mathrm{t}$ (Student) y de permanencia (Helmert) para análisis de homogeneidad de las series. Finalmente, el método de correlación de Spearman se utilizó para evaluar diferencias estadísticas entre rangos de datos para cada estación en particular. 


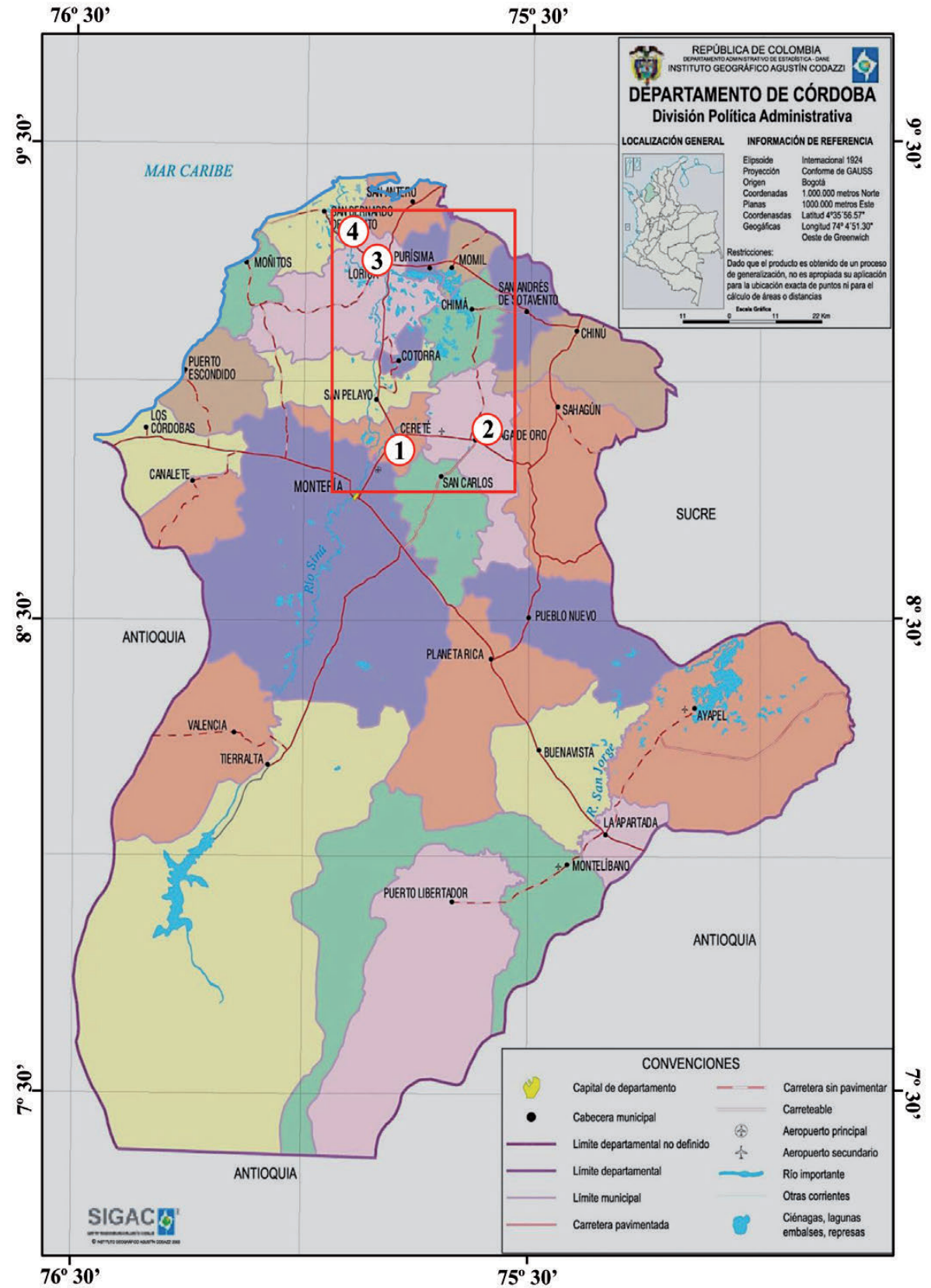

Figura 1. Ubicación geográfica de la zona de estudio (rectángulo en rojo) y localización de las estaciones meteorológicas seleccionadas (1: Turipaná, 2: El Salado, 3: Lorica y 4: La Doctrina). 
Para la generación de datos ausentes de los promedios mensuales de $\mathrm{T}$ (3\% a 7\% según estación meteorológica) se realizó un análisis de regresión simple lineal entre pares de estaciones, coeficientes de correlación $r>0.75$, utilizando en cada caso la serie de datos de la estación Turipaná como variable independiente, por ser la que exhibió la totalidad y la mayor calidad de los datos. La serie de promedios mensuales de HR (12\% a $20 \%$ de datos ausentes) se completó utilizando la media móvil entre ellos. Como validación de la metodología de completado de datos se aplicaron correlaciones de Spearman y simple lineal a todas las combinaciones posibles de los pares de series de datos de las diferentes estaciones. Posteriormente y a partir de las series de datos completadas (promedios mensuales), se calcularon nuevas series de promedios anuales de T y HR, las cuales luego de pasar nuevamente por el proceso de calidad descrito anteriormente, se utilizaron para la identificación y análisis del fenómeno del calentamiento global en la zona de estudio, para todo el periodo de tiempo definido y por décadas.

Debe resaltarse que también se utilizaron series de datos de la precipitación (acumulados anuales), disponibles entre 1971 y 2010, las cuales luego de haber sido verificada su calidad estadística y completadas por medio de los métodos mencionados anteriormente, se utilizaron para el análisis de las tendencias interanuales.

\subsection{Cálculo de la densidad de vapor de agua atmosférica}

Para abordar el estudio del fenómeno del calentamiento global en la zona de estudio, se analizaron inicialmente los comportamientos climáticos de $\mathrm{T}$ y HR, durante el periodo de tiempo seleccionado, calculándose posteriormente la densidad del vapor de agua atmosférica a nivel de la caseta meteorológica $\mathrm{d}_{\mathrm{v}}$, de acuerdo a la ecuación termodinámica para los gases ideales (Linke y Baur, 1970):

$$
d_{v}=\frac{t_{v}}{R_{v} T}
$$

donde, $\mathrm{T}$ es la temperatura del aire en grados Kelvin, $\mathrm{R}_{\mathrm{v}}$ es la constante específica del vapor de agua $461.8=\frac{J}{k g K} \mathrm{y} \mathrm{t}_{\mathrm{v}}$ es la tensión o presión parcial del vapor de agua determinada según Fernández-García (1995):

$$
t_{v}=\frac{H R}{100} \times t_{s}
$$

siendo t la tensión de saturación del vapor de agua (Leckner, 1978),

$$
t_{s}=\exp (26.23-5416 / T)
$$

Los resultados obtenidos para $d_{v}$ también fueron analizados temporalmente entre 1968 y 2011. 


\subsection{Evaluación estadística de tendencias}

Puesto que el fenómeno del calentamiento global se puede interpretar como el resultado del análisis de las tendencias de $\mathrm{T}$ y como se verá más adelante del contenido de vapor de agua atmosférico $d_{v}$, se hizo necesario validar estadísticamente dicho comportamiento. Inicialmente las tendencias interanuales de las series de datos analizadas se ajustaron por medio de la aplicación del filtro de suavizado de Savitzky-Golay, que preserva al máximo los momentos o cambios más importantes de series de datos temporales reduciendo al mismo tiempo en forma significativa el "ruido" de los datos (TableCurve, 2000), y de un análisis de regresión simple lineal (Figs. 3 y 4). Posteriormente, la significancia estadística de las tendencias parciales encontradas se evaluó por medio del cálculo del p-valor y del coeficiente de correlación por rangos $\tau$ de Kendall (Wilks, 2006).

\section{Resultados y discusión}

\subsection{Climatología}

Uno de rasgos más importante del régimen climático de la zona de estudio lo define el comportamiento bimodal, tanto de T como de HR (Fig. 2): mientras que T presenta dos máximos (época seca), el mayor en marzo-abril y luego uno menor en julio, HR los tiene justamente en la época lluviosa: mayo-junio (menor) y septiembre-noviembre (mayor). Temperaturas mínimas son representativas de la temporada lluviosa de cada año en mayo-junio (menor intensidad) y septiembre-noviembre de mayor intensidad, ocurriendo lo contrario con HR: mínimos en época seca en los meses de marzo (mayor) y julio (menor). Otra de las particularidades del clima en las cuatro estaciones seleccionadas, es que la amplitud entre máximos y mínimos para T varía entre $0.8^{\circ} \mathrm{C} \mathrm{y} 1.1^{\circ} \mathrm{C}$, mientras que la variación de HR está entre $77 \%$ y $87 \%$ (Fig. 2). Todas estas características son representativas del clima de la región Caribe colombiana, de acuerdo con IDEAM (2005).

Aunque en numerosos estudios (p. ej., Dai, 2006; Seidel et al., 2007; Brown y DeGaetano, 2013), T y HR son utilizadas comúnmente para la descripción de los cambios climáticos de una zona geográfica, por su simpleza mensurable y facilidad analítica, HR no es la variable termodinámica adecuada para representar las importantes variaciones interanuales del contenido de vapor de agua en la atmósfera. Como se dijo anteriormente, en la zona de estudio seleccionada, HR no muestra variaciones y patrones climáticos significativos, lo que también concuerda con lo expuesto por los autores citados al comienzo de este párrafo, sobre las relativamente pequeñas variaciones interanuales de dicha variable en el Planeta. Por consiguiente, el estudio del fenómeno del calentamiento global en la zona de estudio seleccionada, se centró en las variaciones experimentadas por $\mathrm{T}$ y la variable termodinámica calculada $\mathrm{d}_{\mathrm{v}}$, durante el periodo de tiempo de 44 años (1968-2011).

Antes de comenzar con el análisis de la Fig. 3 y aunque los resultados de los tests aplicados a las series de T, sobre la existencia de datos atípicos, determinaron su validez estadística, fue necesario comprobar si formaban parte del comportamiento climático de la zona de estudio. Puesto que Colombia está bajo la influencia del fenómeno ENSO (IDEAM, 1997; Benavides-Ballesteros y Rocha-Enciso (2012), se comparó cualitativamente la variabilidad interanual de $\mathrm{T}$ (promedios anuales) con el comportamiento tem- 
poral del índice ONI, representativo de los fenómenos climáticos de El Niño y La Niña (NOAA, 2013), encontrándose una estrecha relación entre los valores máximos y mínimos de T con la ocurrencia de cada uno de dichos fenómenos, respectivamente.
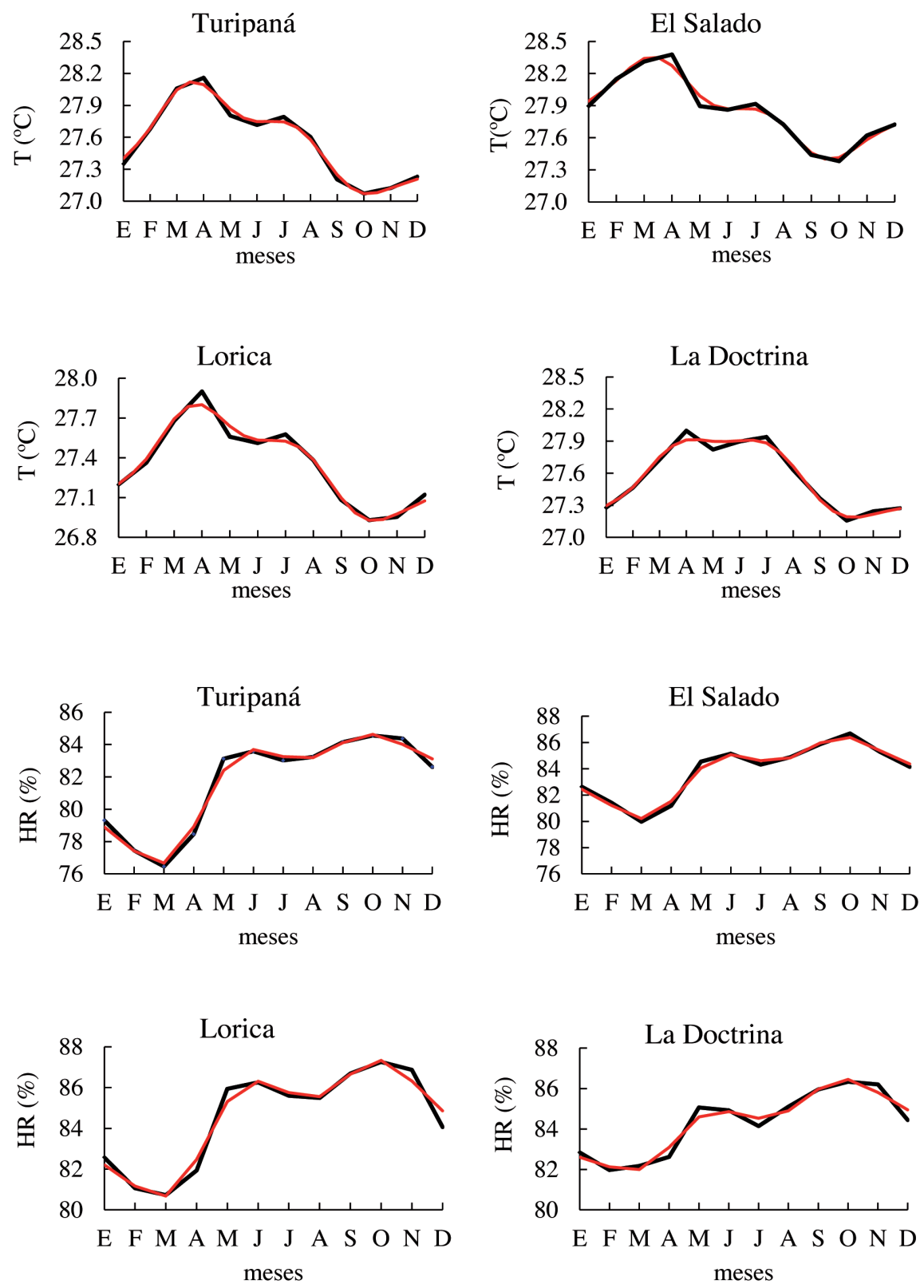

Figura 2. Comportamiento mensual de las series de la temperatura del aire T y humedad relativa RH (promedios mensuales multianuales) de las cuatro estaciones meteorológicas seleccionados (línea roja: filtro de Savitzk-Golay de orden 4). 

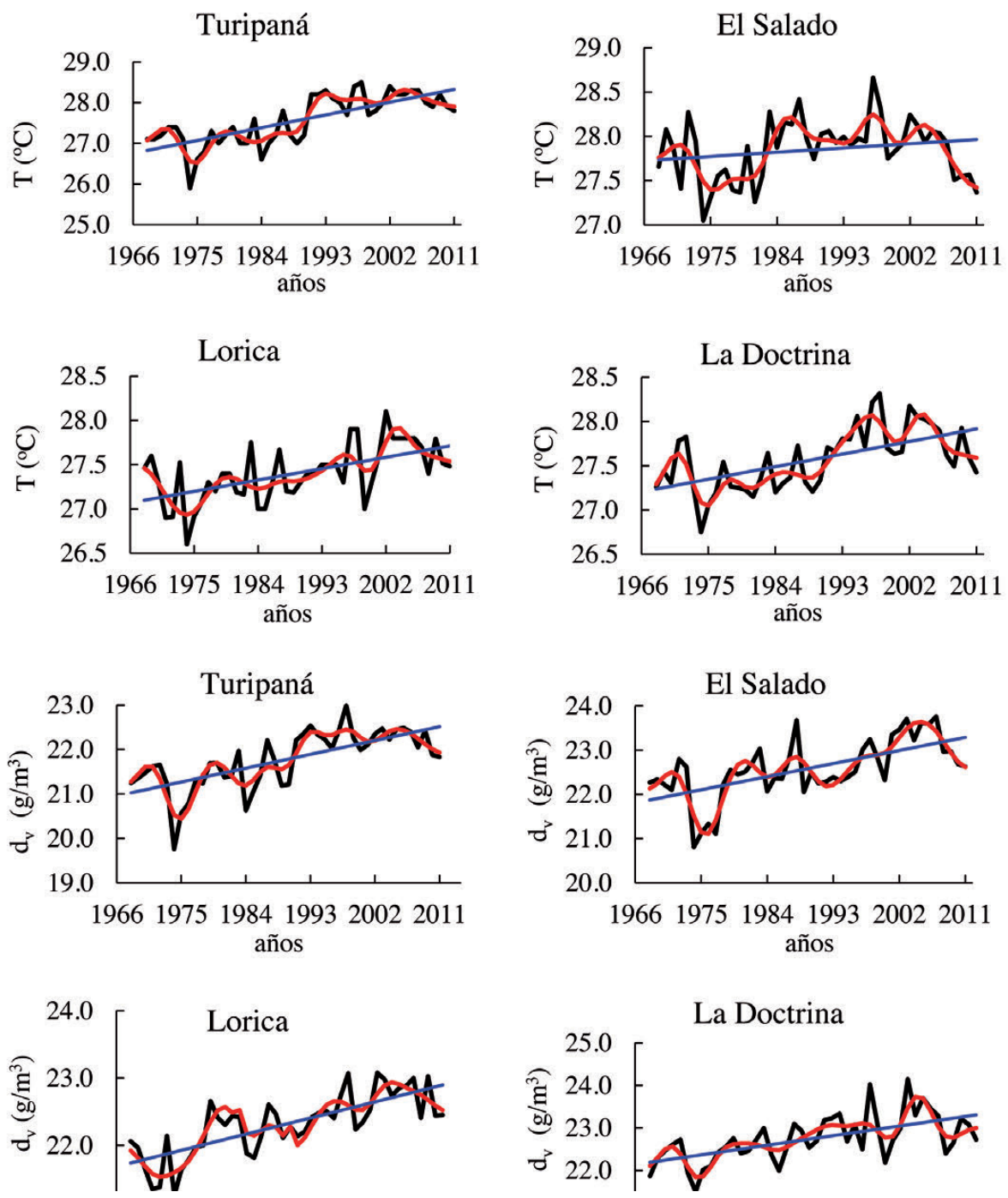

Figura 3. Variación interanual (1968-2011) de la temperatura del aire T y de la densidad de vapor de agua atmosférica $d_{v}$ de las cuatro estaciones meteorológicas seleccionadas (línea roja: filtro de Savitzky-Golay de orden 8, línea azul: tendencia lineal).

Al examinar detalladamente el comportamiento interanual de $\mathrm{T}$ en las estaciones seleccionadas (Fig. 3), se pudo observar que la respectiva serie de datos representa el $77 \%$ de los casos de ocurrencia del fenómeno, tanto de El Niño (aumento de T y ONI entre 0.8 y 2.4): excepto los eventos de 1994-1995, 2004-2005 y 2006-2007, como de La Niña (disminución de T y ONI entre -0.9 y -2.0): excepto los de 1970-1972, 2005-2006 y 2008-2009. El filtro aplicado de Savitzky-Golay, representativo de la tendencia de la serie de datos, también permite identificar los eventos de mayor magnitud de El Niño 
ocurridos en 1968-1970, 1976-1978, el efecto combinado de los eventos entre 1991 y 1998, y 2002-2003, así como también los más intensos de La Niña en los años 1973 1974, 1988-1989, 1998-2001 y la combinación 2007-2010. Al respecto de la ocurrencia de ambos fenómenos es importante anotar que, aunque El Niño ha producido fuertes e intensas sequías en 1982-1983 (IDEAM, 1997) y 1997-1998 (CEPAL, 1999), es La Niña la que origina los mayores efectos catastróficos en la región Caribe colombiana, representados por las grandes y extensas inundaciones de 1974-1975, 1998-2000, 2007-2008 y 2010-2011 (IDEAM, 2011), como consecuencia de fuertes y prolongados períodos de lluvia ocurridos en esta región y en la región andina de Colombia, en donde nacen los principales ríos que desembocan en el mar Caribe.

Como era de esperar, de acuerdo a la ec. (1), $\mathrm{d}_{\mathrm{v}}$ presenta en general el mismo comportamiento que T en todas las estaciones (Fig. 3). Al igual que T, ésta también se muestra sensible a la ocurrencia de ambos fenómenos climáticos globales: $92 \%$ de los eventos El Niño (excepto el de 1994-1995) y 85\% de los de La Niña (excepto 1970-1972 y 2005-2006) se identifican como un aumento y una disminución en el contenido de vapor de agua atmosférico, respectivamente. El filtro aplicado muestra El Niño de 1976-1978, el efecto combinado de los años 1991-1995, 1997-1998 y la combinación 2004-2007, mientras que para La Niña los años 1973-1974 y las combinaciones 1983-1985, 19982001 y $2007-2011$.

\subsection{Tendencias interanuales de $T y d_{v}$}

Actualmente se ha generado un gran debate a nivel mundial sobre la validez de los resultados obtenidos en la tendencia de la temperatura global en las últimas décadas (Antilla, 2005; Lahsen, 2008; Wall Street Journal, 2011; NIPCC-IR1, 2011). Uno de esos estudios, que forma parte de otros con la misma orientación y que se encuentran en NIPCC-IR1 (2011), es el realizado por McLean et al. (2009). En él se afirma que la variabilidad interanual y la tendencia de $\mathrm{T}$ responde fundamentalmente a fenómenos naturales, en particular al ENSO, atribuyéndole muy poca importancia a las actividades antrópicas como una de las posibles causantes del calentamiento global. En contraposición con lo anterior, los científicos adscritos al Working Group I del IPCC (IPCC, 2013) aprobaron el informe que se presentó a la comunidad internacional sobre nuevas evidencias del cambio climático, basadas en múltiples investigaciones (teóricas y observacionales) realizadas sobre el sistema climático mundial. De acuerdo con lo anterior, los resultados de la Fig. 3 muestran que las cuatro estaciones seleccionadas presentan el mismo comportamiento climático reportado por el IPCC (2013), sobre el calentamiento global que actualmente está experimentando el Planeta. Tanto las series multianuales de $\mathrm{T} \mathrm{y} \mathrm{d}_{\mathrm{v}}$, como los filtros aplicados y las tendencias lineales, muestran que desde 1968 estas variables se han incrementado paulatinamente en cada una de las cuatro estaciones.

La cuantificación de los incrementos de T y d entre 1968 y 2011, calculados mediante las ecuaciones de tendencia lineal (Fig. 3: línea azul), significativas estadísticamente, se muestra en la tabla 2. En ella se observa que los incrementos de $\mathrm{T}$ son de diferente magnitud para cada estación, lo cual se debe principalmente a los regímenes de lluvia y de radiación solar existentes en cada una de ellas, así como a la intervención 
antrópica que ha afectado ambientalmente la zona de estudio, representada principalmente por la desecación de humedales (Gónima, 2009). Aunque la zona de estudio ha experimentado aproximadamente la misma clase e intensidad de intervención, excepto en la estación Turipaná en donde ésta ha sido mayor, el menor incremento que muestra T en la estación El Salado $\left(0.23^{\circ} \mathrm{C}\right)$ se debe a que en ese lugar (Tabla 3 ) se registra la mayor cantidad de lluvia en los 40 años de información disponible (1971-2010): 55536 mm acumulados (Turipaná: 51372 mm, Lorica: $52211 \mathrm{~mm}$ y La Doctrina: $52630 \mathrm{~mm}$ ). Una de las características de las regiones tropicales, que además se puso de manifiesto en la discusión anterior sobre el efecto de La Niña, es el hecho de que la lluvia produce una disminución notable de la temperatura del aire, lo cual también es compartido por Trenberth et al. (2005). Contrariamente, el gran incremento de T en la estación Turipaná $\left(1.49^{\circ} \mathrm{C}\right)$ probablemente es la consecuencia del brusco y extensivo cambio en el uso del suelo experimentado por dicho lugar, a partir de los años 1960s, con el fin de desecar pantanos y ciénagas locales para la obtención de terrenos aptos para la ganadería y la agricultura (Pérez-Viloria, 2012). Esta situación ha venido generando la aparición de suelos con poca o nula vegetación y con un gran contenido de agua, que antes no existían, y que al calentarse por efecto de la radiación solar incidente, reflejan e irradian una mayor cantidad de radiación solar y térmica respectivamente, calentando paulatinamente la atmósfera y aumentando al mismo tiempo la evaporación del agua almacenada en ellos. Respecto a los incrementos de $d_{v}$ (Tabla 2), éstos están directamente relacionados con los respectivos aumentos de T para cada estación y con sus causas (precipitación, radiación solar e intervención antrópica). En El Salado, además de la dependencia anterior, al haber más agua disponible en la interfase suelo-vegetación como consecuencia de una mayor cantidad de lluvia precipitada, en comparación con las otras estaciones, es también mayor la cantidad de agua evaporada posteriormente desde la superficie hacia la atmósfera, por efecto de la radiación solar incidente. Finalmente, los datos de la tercera y quinta columnas de la tabla 2, corroboran la mayor sensibilidad y representatividad de $\mathrm{d}_{\mathrm{v}}$ en la descripción del fenómeno del calentamiento global.

Tabla 2. Incremento calculado (tendencia lineal) de la temperatura del aire $T$ y la densidad de vapor de agua $d_{v}$ para cada una de las estaciones meteorológicas (1968-2011).

\begin{tabular}{|c|c|c|c|c|}
\hline Estación & $\boldsymbol{\Delta} \mathbf{T}\left({ }^{\circ} \mathbf{C}\right)$ & $\boldsymbol{\Delta} \mathbf{T}(\boldsymbol{\%})$ & $\boldsymbol{\Delta} \mathbf{d}_{\mathbf{v}}\left(\mathbf{g ~ m}^{-3}\right)$ & $\mathbf{\Delta} \mathbf{d}_{\mathbf{v}}(\boldsymbol{\%})$ \\
\hline Turipaná & 1.49 & 5.5 & 1.48 & 7.0 \\
\hline El Salado & 0.23 & 0.8 & 1.40 & 6.4 \\
\hline Lorica & 0.61 & 2.2 & 1.13 & 5.2 \\
\hline La Doctrina & 0.68 & 2.5 & 1.11 & 4.9 \\
\hline
\end{tabular}

Para confirmar lo expuesto en The Global Climate 2001-2010 (WMO, 2013), respecto a que la década 2001-2010 ha sido considerada la más cálida del periodo 19712010 a nivel mundial, se realizó un análisis cualitativo de la tendencia de $\mathrm{T}$ y d para todas las estaciones (Fig. 3), particularmente en las dos últimas décadas de tiempo (19912000 y 2001-2010). Como resultado, se obtuvo que en la década 1991-2000 se presentó el mayor calentamiento del aire de los 44 años analizados, posiblemente causado por el 
efecto combinado de los fuertes eventos El Niño de 1991-1992 (ONI de 1.6), 1994-1995 (ONI de 1.2) y 1997-1998 (ONI de 2.4), lo que a su vez produjo una disminución notable de las precipitaciones (Tabla 3). En contra a lo que se reporta a nivel global, a partir de 2001 ambas variables presentan tendencia negativa, como consecuencia del enfriamiento de la atmósfera causado por las fuertes y abundantes precipitaciones (Tabla 3), debidas al efecto conjunto de los fenómenos intensos de La Niña de 1998-2001 (ONI de -1.7), 2007-2008 (ONI de -1.5) y 2010-2011 (ONI de -1.5). Estos resultados coinciden con el análisis realizado por Benavides-Ballesteros y Rocha-Enciso (2012) sobre el comportamiento del clima en Colombia (1980-2011) y con estudios realizados a nivel global (p. ej., Dai et al., 2006; Santer et al., 2007; Willet et al., 2007; Isaac y van Wijngaarden, 2012; WMO, 2013).

\subsection{Análisis de promedios decadales de $T y d_{v}$}

Aunque la validez del análisis de la variabilidad climática por medio de promedios decadales está actualmente en discusión, debido a que todavía no está muy claro si los fenómenos climáticos de una zona en particular están bien representados por dicho período de tiempo (Murphy et al., 2010), la Tabla 3 muestra los resultados obtenidos de T $\mathrm{y} \mathrm{d}_{\mathrm{v}}$ en forma decadal.

Tabla 3. Promedios decenales calculados de la temperatura del aire Ty la densidad de vapor de agua $d_{v}$ y precipitación acumulada pp, para cada una de las estaciones meteorológicas.

\begin{tabular}{|c|c|c|c|c|c|}
\hline \multirow{2}{*}{ Estación } & Variable & $\mathbf{1 9 7 1 - 1 9 8 0}$ & $\mathbf{1 9 8 1 - 1 9 9 0}$ & $\mathbf{1 9 9 1 - 2 0 0 0}$ & $\mathbf{2 0 0 1 - 2 0 1 0}$ \\
\hline \multirow{4}{*}{ Turipaná } & $\mathrm{T}\left({ }^{\circ} \mathrm{C}\right)$ & 27.0 & 27.2 & 28.1 & 28.1 \\
\cline { 2 - 6 } & $\mathrm{d}_{\mathrm{v}}\left(\mathrm{g} \mathrm{m}^{-3}\right)$ & 21.1 & 21.4 & 22.3 & 22.3 \\
\cline { 2 - 6 } & $\mathrm{pp}(\mathrm{mm})$ & 11453 & 13179 & 12871 & 13869 \\
\hline \multirow{4}{*}{ El Salado } & $\mathrm{T}\left({ }^{\circ} \mathrm{C}\right)$ & 27.6 & 27.9 & 28.0 & 27.9 \\
\cline { 2 - 6 } & $\mathrm{d}_{\mathrm{v}}\left(\mathrm{g} \mathrm{m}^{-3}\right)$ & 21.9 & 22.6 & 22.7 & 23.3 \\
\cline { 2 - 6 } & $\mathrm{pp}(\mathrm{mm})$ & 13433 & 13909 & 13233 & 14961 \\
\hline \multirow{4}{*}{ Lorica } & $\mathrm{T}\left({ }^{\circ} \mathrm{C}\right)$ & 27.1 & 27.2 & 27.5 & 27.7 \\
\cline { 2 - 6 } & $\mathrm{d}_{\mathrm{v}}\left(\mathrm{g} \mathrm{m}^{-3}\right)$ & 21.9 & 22.2 & 22.5 & 22.8 \\
\cline { 2 - 6 } & $\mathrm{pp}(\mathrm{mm})$ & 11254 & 14056 & 12450 & 14451 \\
\hline \multirow{3}{*}{ La Doctrina } & $\mathrm{T}\left({ }^{\circ} \mathrm{C}\right)$ & 27.3 & 27.4 & 27.9 & 27.9 \\
\cline { 2 - 6 } & $\mathrm{d}_{\mathrm{v}}\left(\mathrm{g} \mathrm{m}^{-3}\right)$ & 22.3 & 22.6 & 23.0 & 23.2 \\
\cline { 2 - 6 } & $\mathrm{pp}(\mathrm{mm})$ & 11785 & 13832 & 12939 & 14074 \\
\hline
\end{tabular}

De acuerdo con la Tabla 3, entre 1971 y 2000 todas las estaciones registran un aumento continuo de $\mathrm{T} \mathrm{y} \mathrm{d}_{\mathrm{v}}$, corroborándose igualmente que la década 1991-2000 fue la más cálida de todas. Esta tendencia se interrumpe durante 2001-2010, al no presentar ambas variables un comportamiento similar en todas las estaciones, haciendo imposible identificar un patrón climático común a ellas durante esta década y contradiciendo además lo expuesto en la sección 4.2, relativo a que $\mathrm{T} \mathrm{y} \mathrm{d}$ muestran una tendencia 
negativa en dicho periodo en comparación con la década inmediatamente anterior. Los incrementos de $\mathrm{T} \mathrm{y} \mathrm{d}_{\mathrm{v}}$ calculados a partir de los datos de la tabla 3, entre las décadas $1971-1980$ y $2001-2010$, fueron de $1.1^{\circ} \mathrm{C}\left(0.27^{\circ} \mathrm{C} \mathrm{dec}^{-1}\right)$ y $1.2 \mathrm{~g} \mathrm{~m}^{-3}\left(0.30 \mathrm{~g} \mathrm{~m}^{-3} \mathrm{dec}^{-1}\right)$ para Turipaná, $0.3^{\circ} \mathrm{C}\left(0.07^{\circ} \mathrm{C} \mathrm{dec}{ }^{-1}\right)$ y $1.4 \mathrm{~g} \mathrm{~m}^{-1}\left(0.35 \mathrm{~g} \mathrm{~m}^{-3} \mathrm{dec}^{-1}\right)$ para El Salado, y $0.6^{\circ} \mathrm{C}$ $\left(0.15^{\circ} \mathrm{C} \mathrm{dec}{ }^{-1}\right)$ y $0.9 \mathrm{~g} \mathrm{~m}^{-3}\left(0.22 \mathrm{~g} \mathrm{~m}^{-3} \mathrm{dec}^{-1}\right)$ para Lorica y La Doctrina. Estos últimos resultados, que además difieren de los obtenidos con las ecuaciones de tendencia lineal (Tabla 2), confirman la gran diversidad en la variabilidad climática de cada uno de los lugares en donde se encuentra cada estación meteorológica, lo cual es característico de climas locales.

\subsection{Evidencias del posible inicio del calentamiento global}

Los registros históricos (1951-2012) de la temperatura superficial del mar en el Pacífico Central (NOAA, 2013) muestran que entre 1973 y 1974 se presentó el fenómeno de La Niña más intenso de toda la historia (ONI de -2.0). Al observar detalladamente la Fig. 3 se comprueba que efectivamente éste, para todas las estaciones, estuvo caracterizado por las más bajas $\mathrm{T} \mathrm{y} \mathrm{d}_{\mathrm{v}}$ de todo el periodo de tiempo estudiado (44 años), identificándose al mismo tiempo en las respectivas series de datos, dos comportamientos temporales claramente opuestos: mientras que entre 1968 y 1974 la tendencia de ambas variables es negativa (disminución), a partir de 1975 ésta se invierte (aumento), manteniéndose así hasta 2011. Con el fin de asegurarse que el comportamiento de ambas tendencias parciales no era el resultado de "artefactos" o del simple azar, se realizó un riguroso análisis estadístico, representado por la aplicación de tests de significancia del p-valor y del coeficiente de correlación $\tau$ de Kendall (Tabla 4).

Tabla 4. Validación estadística del comportamiento temporal parcial de $T y d_{v}$ (1968-1974 y 1975-2011).

\begin{tabular}{|c|c|c|c|c|}
\hline \multirow{2}{*}{ Estación } & \multicolumn{2}{|c|}{$\mathbf{T}$} & \multicolumn{2}{c|}{$\mathbf{d}_{\mathbf{v}}$} \\
\cline { 2 - 5 } & $\mathbf{p}_{(\mathbf{0 . 0 5})}$ & $\tau$ & $\mathbf{p}_{(\mathbf{0 . 0 5})}$ & $\tau$ \\
\hline Turipaná & 0.000370 & 0.0457 & 0.001213 & 0.0849 \\
\hline El Salado & 0.080652 & 0.0457 & 0.0049411 & 0.1503 \\
\hline Lorica & 0.01389 & 0.0196 & 0.0000116 & 0.3464 \\
\hline La Doctrina & 0.012337 & 0.0588 & 0.0001371 & 0.2156 \\
\hline
\end{tabular}

Los tests aplicados (Tabla 4) demuestran que estadísticamente ambas tendencias parciales (1968-1974 y 1975-2011), presentes en cada una de las series de $\mathrm{T} \mathrm{y} \mathrm{d}$, son significativamente diferentes, al estar todos los p-valores y $\tau$ coeficientes por debajo del umbral de 0.05 y 0.61 (Förster y Rönz, 1979), respectivamente. Aunque la serie de datos parcial 1968-1974 es muy corta, debido a la carencia de información meteorológica confiable anterior a 1968, los resultados obtenidos evidencian el cambio abrupto en el comportamiento temporal de ambas variables, entre 1974 y 1975. Por consiguiente, existe la posibilidad de que justamente después del fuerte evento de La Niña (1973-1974), se desencadenó el fenómeno del calentamiento global en la zona geográfica definida por las estaciones meteorológicas seleccionadas. 


\subsection{Análisis integrado de la zona de estudio}

Como se expuso en la sección 2, las cuatro estaciones meteorológicas seleccionadas son representativas del régimen climático de la zona de estudio. Por consiguiente, el comportamiento climático integrado de dicha zona se analizó a partir del cálculo de nuevas series de $\mathrm{T} \mathrm{y} \mathrm{d}_{\mathrm{v}}$ (1968-2011), obtenidas promediando los respectivos valores de las cuatro estaciones (Fig. 4).
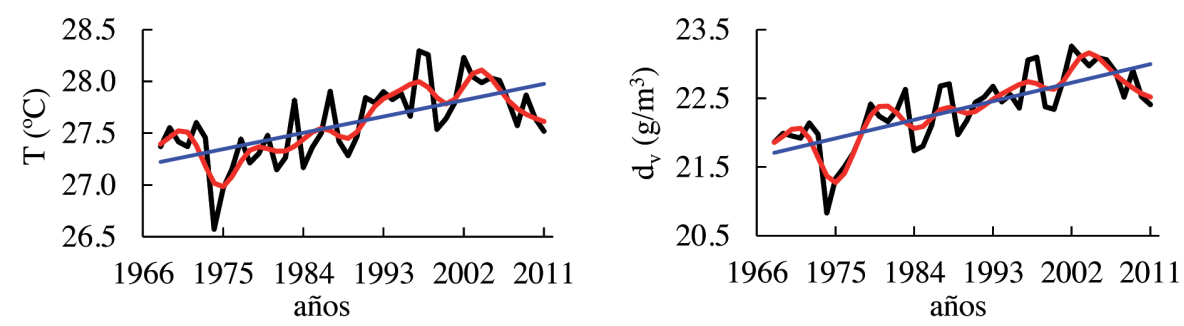

Figura 4. Variación interanual (1968-2011) de la temperatura del aire T y de la densidad de vapor de agua atmosférico dv de la zona de estudio integrada (línea roja: filtro de Savitzky-Golay de orden 8, línea azul: tendencia lineal).

El comportamiento de los promedios decadales de $\mathrm{T} \mathrm{y} \mathrm{d}_{\mathrm{v}}$, en las cuatro décadas en las que se dividió el periodo de tiempo entre 1971 y 2010, es el mismo que el descrito para las cuatro estaciones (sección 4.3): entre 1971 y 1990, T aumentó en $0.1^{\circ} \mathrm{C}$, mientras que $\mathrm{d}_{\mathrm{v}}$ lo hizo en $0.4 \mathrm{~g} \mathrm{~m}^{-3}$. El mayor incremento de $\mathrm{T} \mathrm{y} \mathrm{d}$ ocurrió entre las décadas $1981-1990$ y $1991-2000$ y fue de $0.4^{\circ} \mathrm{C}$ y $0.5 \mathrm{~g} \mathrm{~m}^{-3}$ respectivamente, siendo 1991-2000 la década con el mayor calentamiento de todas. Contrariamente a los resultados obtenidos a nivel mundial (WMO, 2013), en la década 2001-2010, tanto $\mathrm{T}_{\text {como }} \mathrm{d}_{\mathrm{v}}$ no muestran ninguna variación respecto al periodo anterior $\left(27.8^{\circ} \mathrm{C}\right.$ y $\left.22.6 \mathrm{~g} \mathrm{~m}^{-3}\right)$, confirmando lo ya expuesto sobre la aplicación de promedios decadales al estudio de la variabilidad climática tropical a escala local. Además, si se considera todo el periodo de tiempo mencionado (1971-2010), T se incrementó en $0.5^{\circ} \mathrm{C}$, lo que corresponde a un aumento lineal de $0.125^{\circ} \mathrm{C} \mathrm{dec}^{-1}$, valor sensiblemente menor que los $0.17^{\circ} \mathrm{C} \mathrm{dec}{ }^{-1}$ reportado por la WMO (2013) a nivel mundial. En ese mismo periodo de tiempo $\mathrm{d}_{\mathrm{v}}$ aumentó en $0.8 \mathrm{~g} \mathrm{~m}^{-3}$, equivalente a $0.20 \mathrm{~g} \mathrm{~m}^{-3} \mathrm{dec}^{-1}$. Estos resultados confirman lo expuesto en la sección 4.3, sobre la deficiencia de los promedios decadales para representar adecuadamente el comportamiento climático de zonas como la seleccionada en este estudio.

Según la Fig. 4, la serie de datos de T permite identificar el $85 \%$ de los eventos El Niño y La Niña, mientras que en $d_{v}$ se reconocen el $92 \%$ de dichos fenómenos. En cuanto a las tendencias interanuales se observa el mismo comportamiento descrito en la Fig. 3 para las cuatro estaciones meteorológicas: aumento continuo de $\mathrm{T} \mathrm{y}_{\mathrm{v}}$ entre 1968 y 2011. Al igual que en la sección 4.2, el análisis particular de las dos últimas décadas (1991-2000 y 2001-2010), tanto para T como para $\mathrm{d}_{\mathrm{v}}$, muestra que en la década 1991-2000 se registraron los valores más altos de ambas variables en los 44 años ana- 
lizados. Aunque estas variables tendieron a la baja en 2001-2010, en comparación con la década anterior, la tendencia global al aumento de ambas se mantiene desde 1968. Al calcular los incrementos de ambas variables, por medio de las respectivas ecuaciones de tendencia lineal (significativas estadísticamente), se obtuvo que en los 44 años $\mathrm{T}$ ha aumentado en $0.75^{\circ} \mathrm{C}(2.7 \%)$, valor que representa un incremento de $0.17^{\circ} \mathrm{C} \mathrm{dec}^{-1} \mathrm{y}$ que es igual al expuesto en WMO (2013) para el calentamiento global decadal del Planeta (1971-2010), mientras que $\mathrm{d}_{\mathrm{v}}$ lo ha hecho en $1.02 \mathrm{~g} \mathrm{~m}^{-3}$ (5.9\%) ó $0.23 \mathrm{~g} \mathrm{~m}^{-3} \mathrm{dec}^{-1}$. Como se puede observar, el aumento de $\mathrm{d}_{\mathrm{v}}$ es mayor porcentualmente que el experimentado por $\mathrm{T}$, lo cual tiene importantes consecuencias en el régimen climático de la zona de estudio, ya que al aumentar T en el transcurso del tiempo, mayor será el incremento del contenido de vapor de agua en la atmósfera. Finalmente, la Fig. 4 también evidencia la posible iniciación del fenómeno del calentamiento global en la zona de estudio, entre 1974 y 1975, lo que fue verificado estadísticamente al obtener un p-valor y un $\tau$ de Kendall, tanto para $\mathrm{T}(0.00296$ y 0.0065$)$ como para $\mathrm{d}_{\mathrm{v}}(0.00034$ y 0.2549$)$, por debajo de los umbrales definidos en la sección 4.4.

\section{Conclusiones}

Los resultados obtenidos, luego de haber sometido las series de datos de la temperatura del aire y de la humedad relativa a un exhaustivo análisis estadístico sobre su calidad, confirman que, aunque fenómenos atmosféricos y oceánicos como El Niño y La Niña modifican parcialmente el clima de la zona de estudio en determinados años, éstos no interrumpieron la tendencia al calentamiento que se experimenta en ella desde 1968 y que posiblemente sea la consecuencia de la extensa e intensa intervención antrópica que experimenta esta zona desde los años 1960s (desecación de humedales). Este fenómeno, para la zona de estudio integrada, significó un incremento de la temperatura del aire de $0.75^{\circ} \mathrm{C}(1968-2011)$, calculado por medio de la tendencia lineal, lo que a la vez representa un aumento de $0.17^{\circ} \mathrm{C}$ por década, valor que es igual a la tasa de cambio lineal reportada por la Organización Meteorológica Mundial sobre el calentamiento global que actualmente está ocurriendo a nivel mundial.

De acuerdo con las ecuaciones termodinámicas utilizadas para el cálculo de la densidad de vapor de agua atmosférico, el comportamiento de ésta en la zona de estudio (aumento o disminución) es equivalente al de la temperatura del aire. Además y según todos los análisis realizados, tanto para cada una de las estaciones meteorológicas seleccionadas como para la zona de estudio integrada, la densidad de vapor de agua atmosférica es la variable termodinámica que mejor representa la variabilidad climática y la ocurrencia del calentamiento global. Por ejemplo, para la zona de estudio integrada, mientras la temperatura del aire se incrementó en $2.7 \%$, la densidad de vapor de agua lo hizo en $5.9 \%$, a razón de $0.23 \mathrm{~g} \mathrm{~m}^{-3}$ por década, durante los 44 años estudiados.

El análisis cualitativo de tendencia lineal permitió verificar que la década 19912000 fue la más cálida del período 1968-2011. Esto difiere de lo afirmado por diversos estudios realizados a nivel mundial, respecto a que en la década 2001-2010 se presentaron las temperaturas más altas de todo el período de tiempo seleccionado. En realidad y de acuerdo al mencionado análisis, entre 2001 y 2010 tanto la temperatura del aire como 
el contenido de vapor de agua atmosférico muestran una tendencia negativa, lo cual concuerda con otros estudios realizados sobre la variabilidad climática de Colombia durante esa década. Estos resultados confirman la importancia que tienen, para el análisis de la variabilidad climática, las particularidades locales de cada una de las estaciones meteorológicas analizadas, así como las de la zona de estudio integrada.

A diferencia de la profusión de estudios realizados a escalas global y regional sobre el fenómeno del calentamiento global, en los cuales los promedios decadales contribuyen a explicar el comportamiento climático de las variables involucradas, los resultados obtenidos con ellos en esta investigación, aunque también permitieron identificar a la década 1991-2000 como la de mayor calentamiento, contradicen el comportamiento que presentan en realidad la temperatura del aire y la densidad de vapor de agua atmosférico entre 1991-2000 y 2001-2010. Para la zona de estudio, los promedios decadales no pueden representar completamente la gran variabilidad climática local, la cual además está fuertemente influenciada por los fenómenos El Niño y La Niña.

Los resultados del análisis estadístico realizado a las series de datos de la temperatura del aire y la densidad de vapor de agua atmosférica para los períodos 1968-1974 y 1975-2011, podrían interpretarse como una evidencia de que el posible inicio del calentamiento global en la zona de estudio, está estrechamente relacionado con la ocurrencia del más intenso evento de La Niña de la historia, sucedido entre 1973 y 1974. Futuros estudios en otras zonas de la región Caribe colombiana servirán para confirmar estos resultados.

\section{Referencias bibliográficas}

Aro, T.O. 1976. Analysis of data on surface and tropospheric water vapour. Journal of Atmospheric and Terrestrial Physics 38, 565-57.

Antilla, L. 2005. Climate of scepticism: US newspaper coverage of the science of climate change. Global Environmental Change 15, 338-352.

Benavides-Ballesteros, H.O, Rocha-Enciso, C.E 2012. Indicadores que manifiestan cambios en el sistema climático de Colombia (Años y décadas más calientes y las más y menos lluviosas). IDEAM-METEO/001-2012, Nota Técnica del IDEAM, Instituto de Hidrología, Meteorología y Estudios Ambientales, Bogotá, 26 pp.

Brown, P.J., DeGaetano, A.T. 2013. Trends in U.S. surface humidity, 1930-2010. Journal of Applied Meteorology and Climatolology 52, 147-163.

Comisión Económica para América Latina y el Caribe (CEPAL) 1999. Efectos macroeconómicos del fenómeno El Niño de 1997-1998. LC/MEX/R.688, Comisión Económica para América Latina y el Caribe, 38 pp.

Corporación Autónoma Regional de los Valles del Sinú y San Jorge (CVS), Parques Nacionales Naturales de Colombia (PNN), Corporación Autónoma Regional de Sucre (CARSUCRE) y Universidad de Córdoba 2006. Fases de prospección y formulación del Plan de Ordenamiento y Manejo Integral de la Cuenca Hidrográfica del Río Sinú(POMCA-RS). Montería, 859 pp.

Dai, A. 2006. Recent climatology, variability, and trends in global surface humidity. Journal of Climate 19, 3589-3606.

Fernández-García, F. 1995. Manual de climatología aplicada. Síntesis, Madrid, 385 pp.

Förster, E., Rönz, B. 1979. Methoden der Korrelations- und Regressionsanalyse. Verlag Die Wirtschaft, Berlin, 324 pp. 
Gónima, L. 2009. Evidencias del calentamiento global en el Caribe Colombiano. Meteorología Colombiana 13, 1-10.

Instituto de Hidrología, Meteorología y Estudios Ambientales (IDEAM) 1997. Posibles efectos naturales y socioeconómicos del fenómeno El Niño en el período 1997-1998 en Colombia. Bogotá, $71 \mathrm{pp}$.

Instituto de Hidrología, Meteorología y Estudios Ambientales (IDEAM) 2005. Atlas Climático de Colombia. Bogotá, 219 pp.

Instituto de Hidrología, Meteorología y Estudios Ambientales (IDEAM) 2011. Boletín informativo sobre el monitoreo del fenómeno de "La Niña". Boletín número 30, Bogotá, 5 pp.

Instituto Geográfico Agustín Codazzi (IGAC) 2009. Estudio general de suelos y zonificación de tierras del Departamento de Córdoba 1:100.000. Bogotá, 502 pp.

Intergovernmental Panel on Climate Change (IPCC) 2001. Cambio Climático 2001: Impactos, adaptación y vulnerabilidad-Contribución del grupo de trabajo II al Tercer informe de evaluación del IPCC 2001. Ginebra, 101 pp.

Intergovernmental Panel on Climate Change (IPCC) 2013. Summary for policymakers. Disponible en: http://www.climatechange2013.org/images/uploads/WGIAR5-SPM_ Approved27Sep2013.pdf (fecha de acceso 11/10/2013).

Isaac, V., van Wijngaarden, W.A. 2012. Water vapor pressure and temperature trends in North American during 1948-2010. Journal Climate 25, 3599-3609.

Kousari, M.R., Ekhtesasi, M.R., Tazeh, M., Saremi-Naeini, M.A., Asadi-Zarch M.A. 2011. An investigation of the Iranian climatic changes by considering the precipitation, temperature, and relative humidity parameters. Theoretical and Applied Climatology 103, 321-335.

Lahsen, M. 2008. Experiences of modernity in the greenhouse: a cultural analysis of a physicist "trio" supporting the backlash against global warming. Global Environmental Change 18, 204-219.

Leckner, B. 1978. The spectral distribution of solar radiation at the earth's surface-elements of a model. Solar Energy 20, 143-150.

León-Aristizabal, G.E. 2000. Tendencia de la temperatura del aire en Colombia. Meteorología Colombiana 2, 57-65.

Linke, F., Baur, F. 1970. Meteorologisches Taschenbuch. Geest \& Portig, Leipzig, 712 pp.

McLean, J.D., de Freitas, C.R., Carter, R.M. 2009. Influence of the Southern Oscillation on tropospheric temperature. Journal of Geophysical Research 114. 10.1029/2008JD011637.

Moeller-Houborg, R., Soegaard, H. 2004. Regional simulation of ecosystem $\mathrm{CO}_{2}$ and water vapor exchange for agricultural land using NOAA AVHRR and Terra MODIS satellite data. Application to Zealand, Denmark. Remote Sensing of Environment 93, 150-167.

Murphy, J., Kattsov, V., Keenlyside, N., Kimoto, M., Meehl, G., Mehtaf, V., Pohlmann, H., Scaife, A., Smith, D. 2010. Towards prediction of decadal climate variability and change. Procedia Environmental Sciences 1, 287-304.

Nongovernmental International Panel on Climate Change (NIPCC-IR1) 2011. Climate Change Reconsidered: 2011 Interim Report of the Nongovernmental International Panel on Climate Change (NIPCC). The Heartland Institute, Chicago, $416 \mathrm{pp}$

National Oceanic and Atmospheric Administration (NOAA) 2013. ENSO cycle: recent evolution, current status and predictions. Disponible en: http://www.cpc.ncep.noaa.gov/ products/analysis_monitoring/lanina/enso_evolution-status-fcsts-web.pdf (fecha de acceso: 13/09/2013).

Ocampo-López, O.L. 2012. Análisis de vulnerabilidad de la cuenca del río Chinchiná para condiciones estacionarias y de cambio climático. Tesis de maestría, Departamento de Ingeniería Química, Universidad Nacional de Colombia-Sede Manizales, Manizales, 255 pp. 
Organización Meteorológica Mundial (OMM) 2008. Adaptación a la variabilidad del clima y al cambio climático. Boletín 57 (2), Ginebra, 68 pp.

Padilla-Agámez, M.H. 2011. Definición de indicadores de cambio climático para la cuenca baja del río Sinú: Distrito de Riego de la Doctrina. Tesis de maestría, Facultad de Ciencias Contables, Económicas y Administrativas, Universidad de Manizales, Manizales, 45 pp.

Pérez-Viloria, M. 2012. Nuevas evidencias del calentamiento global en el Caribe colombiano. En El Cambio Global: su expresión e impactos en Colombia, Memorias en CD del X Ciclo Anual de Conferencias. Departamento de Geografía, Universidad Nacional de Colombia, Bogotá.

Ruiz-Murcia, J.F. 2010. Cambio Climático en temperatura, precipitación y humedad relativa para Colombia usando modelos meteorológicos de alta resolución (panorama 20112100). IDEAM-METEO/005-2010, Nota Técnica del IDEAM, Instituto de Hidrología, Meteorología y Estudios Ambientales, Bogotá, 91 pp.

Santer, B.D., Mears, C., Wentz, F.J., Taylor, K.E., Gleckler, P.J., Wigley, L.T.M., Barnett, T.P., Boyle, J.S., Brüggemann, W., Gillett, N.P., Klein, S.A., Meehl, G.A., Nozawa, T., Pierce, D.W., Stott, P.A., Washington, W.M., Wehner, M.F. 2007. Identification of human-induced changes in atmospheric moisture content. Proceedings of the National Academy of Sciences of the United States of America 39, 15248-15253.

Seidel, Th.M., Grant, A.N., Pszenny, A.A.P., Allman, D.J. 2007. Dewpoint and humidity measurements and trends at the summit of Mount Washington, New Hampshire, 1935-2004. Journal of Climate 20, 5629-5641.

TableCurve 2000. TableCurve 2D 5.0 Automated Curve Fitting \& Equation Discovery. AISN Software Inc.

Trenberth, K.E., Fasullo, J., Smith, L. 2005. Trends and variability in column-integrated atmospheric water vapor. Climate Dynamics 24, 741-758.

United Nations Framework Convention on Climate Change (UNFCCC) 1992. Convención marco de las Naciones Unidas sobre el cambio climático. Disponible en: http://unfccc.int/resource/ docs/convkp/convsp.pdf (fecha de acceso: 13/09/2013).

Wall Street Journal 2011. The case against global-warming skepticism. Opinion Europe. Disponible en: http://online.wsj.com/news/articles/SB100014240529702044224045765948727963273 48 (fecha de acceso: 21/09/2013).

Wilks, D.S. 2006. Statistical methods in the atmospheric sciences. Academic Press, USA, 627 pp.

Willett, K.M., Williams, Jr.C.M., Dunn, R.J.H., Thorne, P.W., Bell, S., de Podesta, M., Jones, P.D., Parker, D.E. 2013. HadISDH: an updateable land surface specific humidity product for climate monitoring. Climate of the Past 9, 657-677.

World Meteorological Organization (WMO) 2011. Guide to climatological practices. WMO-No. 100. Geneva, 115 pp.

World Meteorological Organization (WMO) 2013. The global climate 2001-2010: a decade of climate extremes. WMO-No. 1103. Geneva, $117 \mathrm{pp}$. 